\title{
Analytical model of nonlinear noise in the b-modulated optical transmission systems
}

\author{
Stanislav Derevyanko ${ }^{1}$, Dmitry Shepelsky ${ }^{2}$, Maryna Pankratova ${ }^{3}$, Anastasiia \\ Vasylchenkova $^{3}$, Nikolai Chichkov ${ }^{3}$, and Jaroslaw Prilepsky ${ }^{3}$ \\ ${ }^{1}$ School of Electrical and Computer Engineering, Ben Gurion University of the Negev, Beer Sheva 84105, Israel \\ 2 B.I. Verkin Institute of Low Temperature Physics and Technology, Kharkiv, 61103, Ukraine \\ ${ }^{3}$ Aston Institute of Photonic Technologies, Aston University, Birmingham, B4 7ET, UK. \\ stasd@bgu.ac.il
}

\begin{abstract}
:
We propose an analytical channel model for optical communication systems employing the nonlinear division multiplexing scheme using $b$-modulation and show that as the energy of the NFT burst grows, the noise in the $b$-coefficient decreases.

(C) 2020 The Author(s)
\end{abstract}

\section{Introduction}

The nonlinear Fourier transform (NFT) is a recent promising method aimed at mitigating the simultaneous influence of both chromatic dispersion and Kerr nonlinearity in long haul optical fiber transmission systems [1]. It is based on the notion of the nonlinear spectrum (NS) $r(\xi, z)$, with $\xi$ being the nonlinear analogue of frequency and $z$ is the propagation distance, which generalises its linear Fourier counterpart and evolves as an all-pass filter operation during the nonlinear propagation of the signal in a single-mode optical fiber. The NS can generally contain discrete eigenvalues (solitons) as well, but in the present work, we do not modulate them and deal with the continuous part of the NS only. For simplicity, we shall also limit ourselves to the case of single polarization.

In the NFT-based transmission, a sequence of symbols $c_{k}$ chosen from a conventional modulation constellation is mapped onto $N_{s c}$ subcarriers defined inside the nonlinear Fourier domain:

$$
u_{0}(\xi)=\sqrt{S} \sum_{n=-N_{s c} / 2}^{N_{s c} / 2} c_{k} u(\xi / \Delta \xi-k),
$$

where $\Delta \xi$ is a carrier spacing and $S$ is the power scaling factor. One can identify (1) as the input NS $r(\xi, 0)$, perform the inverse NFT (INFT) operation, and launch our resulting time-domain signal into the fiber. At the receiver side, after coherent detection, the forward NFT is performed and the transmitted symbols $c_{k}$ are recovered by e.g. matched filtering. Recently, a different NFT-based scheme has become popular in which instead of modulating the nonlinear spectrum the so-called $b$-coefficient is modulated. This coefficient has a one-to-one nonlinear relationship to $r(\xi)$ [1-3]. The initial interest to the $b$-modulation was caused by the fact that the band-limited spectral carriers $u(\xi)$ will also have a limited controllable duration in the time domain after applying INFT [2]. The $b$-modulation, however, suffers from the intrinsic constraint: $|b(\xi)|<1$, which in turn limits the maximum energy, $E$, of the $b$-modulated signal. One of the solutions suggested for overcoming this barrier was to use the exponential scaling in mapping our data onto the NS [3]: $b(\xi)=\sqrt{\left(1-\exp \left(-\left|u_{0}(\xi)\right|^{2}\right)\right)} \exp \left(i \angle u_{0}(\xi)\right)$. The advantage of such scaling is that the total energy $E$ is now unbounded and easily controllable by virtue of tuning the parameter $S$. This comes at the expense of a slight loss of the time localisation, but as shown in [3] this loss is not critical. We emphasise that our theoretical results are universal and applicable to other scaling relations; in particular, we also check the theoretical approach for the original linear scaling: $b(\xi)=u_{0}(\xi)[2]$.

\section{Theoretical model}

In the absence of perturbations the $b$ coefficient acquires a deterministic phase shift proportional to the overall distance $L$, expressed here in dimensionless units: $b(\xi, L)=b(\xi, 0) \exp \left(4 i \xi^{2} L\right)$. In the presence of the inline amplifier spontaneous emission noise $N(z, t)$, assumed here as a Gaussian delta-correlated process: $\left\langle N(z, t) N^{*}\left(z^{\prime}, t^{\prime}\right)\right\rangle=$ $2 D \delta\left(z-z^{\prime}\right) \delta\left(t-t^{\prime}\right)$, with $2 D$ being the noise power spectral density, the $b$-coefficient at the receiver after compensating all-pass filtering, acquires a noise induced component $\Delta b(\xi)$. The main theoretical result of this paper is an analytical channel model for the statistics of this NFT noise. Specifically, we show that at high SNR it can be modelled by a symmetric complex white Gaussian noise with the spectra correlation function given by:

$$
\left\langle\Delta b(\xi) \Delta b^{*}\left(\xi^{\prime}\right)\right\rangle=2 \pi D L\left(1-|b(\xi, 0)|^{2}\right) \delta\left(\xi-\xi^{\prime}\right) .
$$


This result was obtained by expanding the perturbative treatment of Ref. [4] to the case of $b$-modulated symbols and is valid when the effective SNR in the NFT domain is large.

\section{Results}

To validate the theoretical predictions of the analytical model (2), we have performed a series of numerical simulations of the full propagation of $N_{s c}=128 \mathrm{~b}$-modulated sinc-shaped carriers down the fiber link with the distributed ASE noise term $N(z, t)$ for single polarization. We have assumed that the symbols were sampled uniformly from a Q-PSK constellation (other constellations yield similar results provided that the average energy of the burst is the same). We have studied the power spectral density (PSD) $B$ of the $b$-coefficient noise normalized by its linear limit $2 \pi D L$ and averaged over input constellation and total nonlinear bandwidth $N_{s c} \Delta \xi$. According to the theoretical prediction (2) this is given by $1-\left\langle|b(\xi, 0)|^{2}\right\rangle$ and is independent on the propagation distance and depends on the input burst energy. However, we noticed that at high energies the processing error of the (I)NFT itself becomes a significant issue and the results presented in Fig. 1 represent the values obtained after subtracting the processing noise obtained via a separate run with the ASE noise switched off.
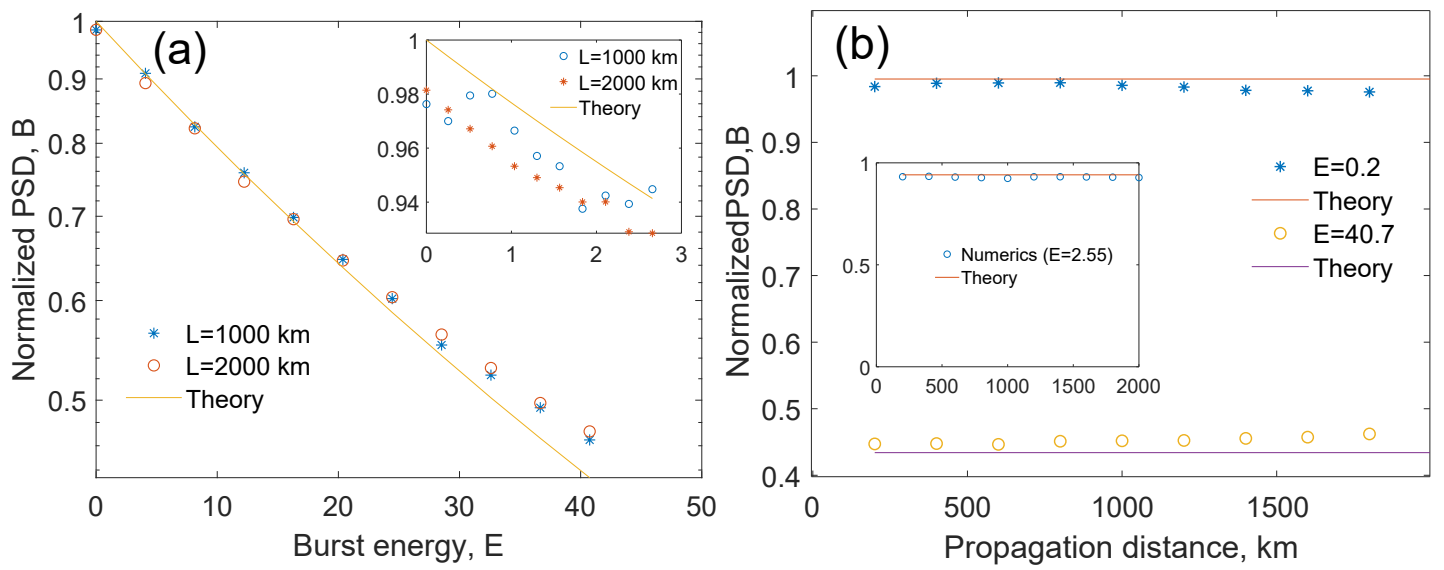

Fig. 1: Dependence of the normalized compensated PSD of the nonlinear spectral noise on the dimensionless burst energy (a) and propagation distance (b). The curves were obtained by averaging over 600 realizations of the input symbols and ASE noise and subtracting the values without ASE. Insets: the same results for the linear scaling scheme utilised, e.g., in Ref. [2]. All numerical results show a good coincidence with the theory.

In all the cases, we have found a good agreement between theory and the numerics for both scaled and unscaled (linear scaling) $b$-modulation with a slight deviation between those at high energies, which we attribute to a high residual processing error (and not to the effect of the ASE).

\section{Conclusion}

We have developed a perturbative analytical model for NFT-based transmission systems employing $b$-modulation. We have shown that the PSD effective noise in the $b$-coefficient is proportional to $1-|b(\xi)|^{2}$ and hence tends to zero when $|b(\xi)|$ reaches its maximum value. This is in stark contrast with the NFT systems employing $r$ modulation [4]. This means that as the burst energy is increased there exists an optimal value achieving a trade-off between the ASE noise and the numerical error.

This project has received funding from the EU Horizon 2020 program under the Marie Sklodowska-Curie Grant Agreement No.751561 (MP), No. 843801 (NC) and from Israeli Science Foundation (grant no 466/18).

\section{References}

1. S.K. Turitsyn et al., "Nonlinear Fourier transform for Optical Data Processing and Transmission: Advances and Perspectives," Optica 3, 307 (2017).

2. S. Wahls, "Generation of Time-Limited Signals in the Nonlinear Fourier Domain via b-Modulation," in Proceedings of European Conference on Optical Communication, paper W.3.C.6, 2017.

3. X. Yangzhang et. al., "Dual Polarization Non-Linear Frequency-Division Multiplexed Transmission With b-Modulation”, J. Lightwave Technol. 37, 1570 (2019).

4. S. Derevyanko et al., "Capacity Estimates for Optical Transmission Based on The Nonlinear Fourier Transform,” Nature Commun. 7, 12710 (2016). 